\title{
Automatic Speech Recognition in Mobile Customer Care Service
}

\author{
Ms. Pooja Sahu \\ Research Scholar, Department of EXTC, KITE Raipur India \\ Poojasahu1991@gmail.com
}

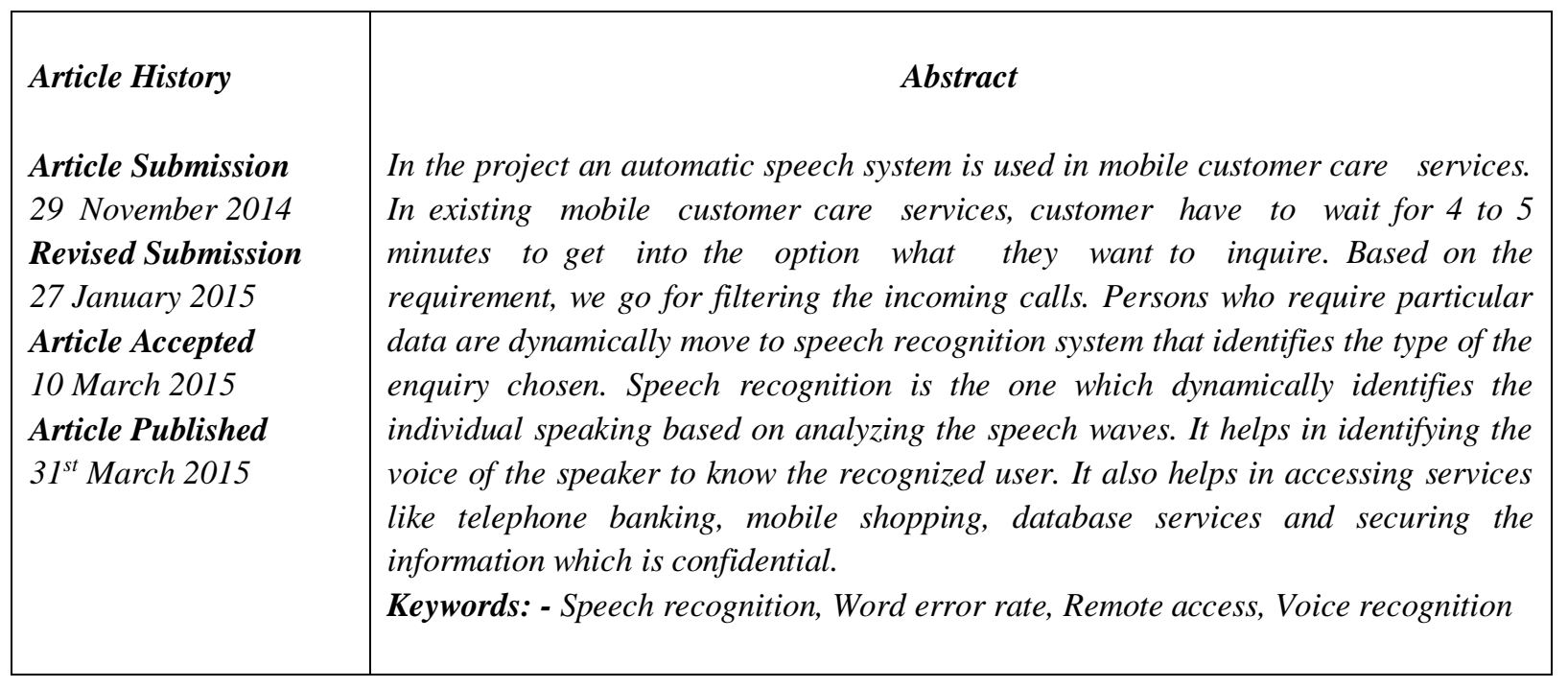

\section{Introduction}

Speech recognition has a benefit of cost and services effectively. Speech recognition performance can be assessed by knowing its speed and accuracy. Here, accuracy means word error rate and speed is nothing but real time velocity. Speech recognition system translates speech into particular user's voice that are trained or employed for authentication or confirms voice of the speaker identity for security purposes [1][2].

Speech recognition which is a dynamic technique generally developed for disabled community. The main purpose of speech recognition system is to convert speech signal into information unconventional to the environment or speech recorded by a system [3]. In general, speech recognition says about the speaker identity, instead of the information that the speaker is speaking. Identifying the speaker makes our duty simpler since the systems have been taught on particular user's voices or employed for authentication or confirms voice of the speaker identity for security purposes. The system model scenario is shown in figure 1.

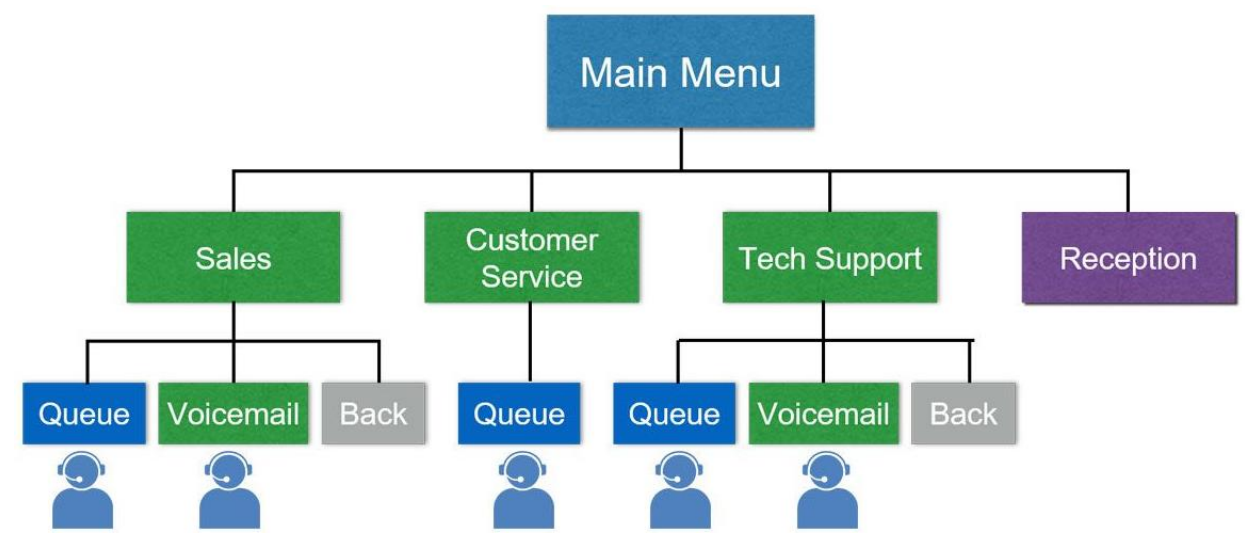

Fig 1: Speech Recognition in Mobile Customer Care Service 
Speech recognition strongly employed to embedded electronic systems such as home automation system, car embedded systems for navigation purposes [4]. ASR is technique to be identified by a computer and perform upon expressions. From the transmitter, the speech is given and in the receiver it directly moves to the specific option. It can be done for all the applications provided by the customer care services. Speech recognition combines Neural Networks and Markovian Models, reducing memory requirements and delivering high levels of accuracy even on large vocabularies [6]. It is speaker independent, but also provides tools for adapting acoustic models with data collected in the field, if required by a particular application. It has the ability to recognize speech, even around large amounts background noise and Reusable, built-in libraries for recognizing addresses, currency values and alphanumeric strings [7].

\section{Related Work}

Interactive voice response is the one which permits a system to link with users via voice signal and input DTMF tones through a keyboard. IVR permits clients to link with company's server system through a keyboard or speech recognition in cellular and mobile communications. Thereafter, they provide service to the enquiries of their own go along with IVR speech. They will respond to automatically developed voice and help the users to move further. Applications of IVR are employed to control any operation in which the computer breaks the whole interaction into smaller parts. Also, they are installed in the network to control volumes of large calls [8].

Also, IVR is employed in automobiles for operating with body parts. Recently automobiles are installed with navigation, audio, video, mobile applications. These are generally employed in telecommunication companies for a dynamic attendance through IVR. Voice over IP professionals and telecommunications professionals generally use the word IVR. Also, voice response is used sometimes [9].

Purposefully IVR systems are employed to provide service to large volumes of calls. It is cost effective. Applications of IVR systems are generally credit card, debit card services (telephone banking). Also, an industry expands their working hours to $24 * 7$ by employing IVR. It also helps the users not to wait in the queue for the queries and reduces the cost of an employee. When the users are not satisfied with the IVR, they are moved to the agents to interact with complex queries. Also, agents pass away yes/no responses.

Call centers employ IVR to segregate called users and identify them. Capability to recognize users permits services which are customized based on their profile. These centres allow the users to stay in the queue or callback request is done. IVR obtains caller line identification information from the database for authentication of the caller. Employing DNIS confirms a right language is simulated in an IVR system which answers different mobile numbers. Only one Huge IVR system holds calls for many applications, each one having its own script. IVR supports polarization in which users will have a status that will dynamically registers the user's call and permits them to move up in the queue. Prioritization is the one which also depends on DNIS [10].

IVR system is also employed by start-ups and small companies to appear their business to be more than what it is. For instance, user never tries to know about their support and sales. Apart from interaction of the users , IVR logs information of the call into the database for billing purpose, usage of the service, advancements in the IVR systems. CTI permits a company to segregate the caller information for moving the enquiry to a preferable agent. It transfers only the most appropriate information of a user and providing effective service. IVR is also employed by survey organizations to be asked by the investigators some thoughtful questions that are bothering them. The user will feel uncomfortable to give away the answers to the agents for questions like usage of drug or behaviour during sex. IVR system limited to some situations may be employed in co-occurrence of a human agent. For instance, the agent may say to the user that for the next call they might be forwarded to the IVR system to stop the inquiry

\section{Proposed Data path Designs}

Push button' IVR is being used in present all customer care service.But in this system user has to wait for long time to get their specified option. Here, speech recognition filters incoming calls based on their inquiries. Also, the users who needs data are dynamically governed to speech recognition system which identifies the feature of the enquiry. There will be a sequence of attempts happened. This can be achieved by getting user voice as an input and converted into text using MATLAB programs and this result is compared with predefined values and then the call is directed to specified option. The proposed flowgraph is shown in figure 2. 


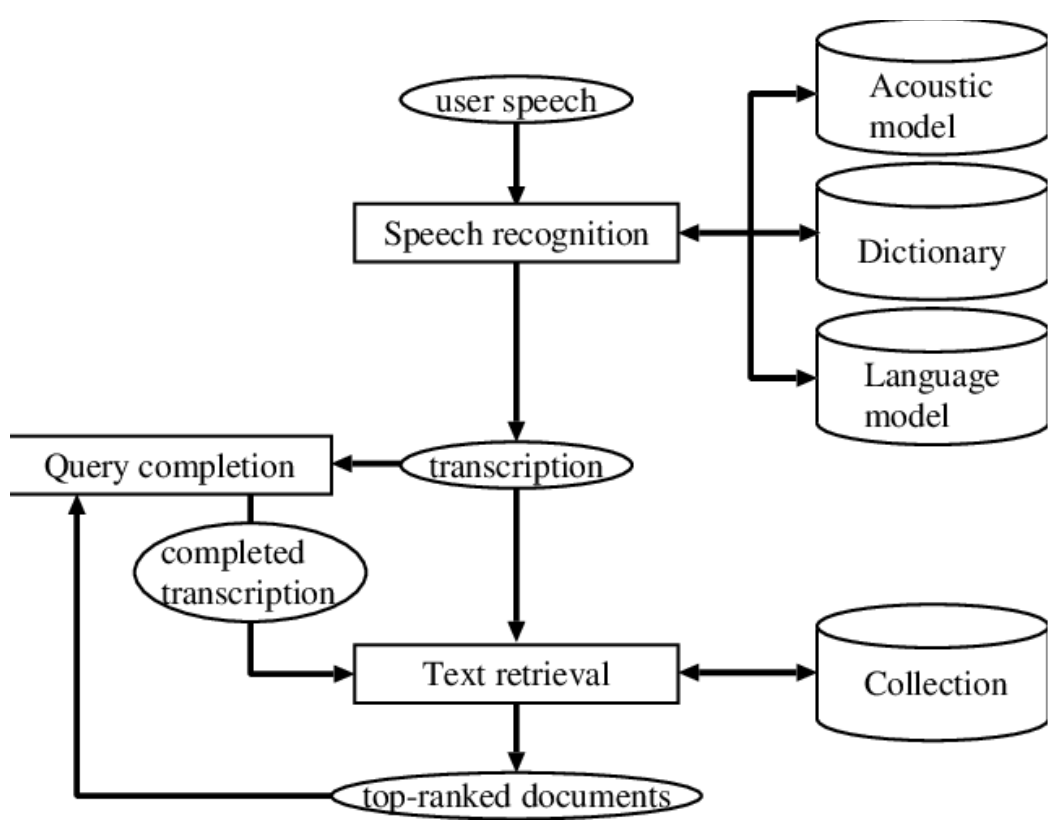

Fig 2: Proposed Speech Recognition in Mobile Customer Care Service

\section{a. Testing Stage}

In this stage the input audio signal from the customer is tested. The tested signal is finally quantized. In the recent directory, program uses the file to display a model in which the files are listed. The dialog box helps the client to choose or open the file by typing a name. Valid filename is present, uigetfile gives back filename when he opens it by clicking. If not, an error is observed and returns back to dialog box. After that, client can go for typing other filename or cancel it. The uigetfile is 0 , when the user cancels the process.

\section{b. Training Stage}

In the training stage the program will reads the database present in the server in terms of the segment, clusters and segments. The main function of the training stage is to calculate the number or length of the audio information present in the database.

\section{c. Comparison Stage}

In the comparison stage, the tested input audio signal is compared with the audio information present in the database using the KNN classification algorithm.

\section{Simulation Results}

$\mathrm{K}-\mathrm{NN}$ algorithm is a specific type which is employed in approximating analogue variables. For instance, we employ an inverse distance of $\mathrm{k}$-nearest different variables having a weighted average. Algorithm functions are discussed below.

a) We have to calculate the distance between Euclidean and target.

b) Samples are taken into consideration for evaluated distances.

c) Heuristic approach is selected for RMSE dependent $\mathrm{k}$ nearest neighbour happened by employing validation process. 


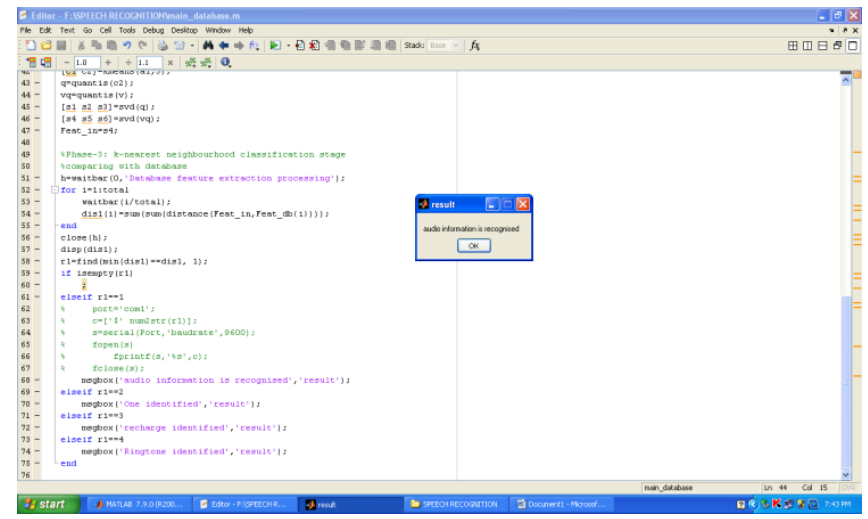

Fig 3: Simulation window showing training stage

Thermal-frequency spectrum coefficients are the one that are combinable to get an MFC in speech processing. These are obtained from a kind of central representation. Compression of an audio or speech in MFFCs is acquired below by the following process. Fourier transform of speech signal is taken into consideration. By employing triangular overlapping windows, we plan spectrum powers on a scale. Each power logs are taken into consideration on mel frequencies. Cosine transform is taken in time domain to list of mel log powers. The amplitudes of MFFCs are the achievements of a spectrum. .The command quant is used to discretize values as multiples of quantity. The MATLAB graphic interface is shown in figure 4 .

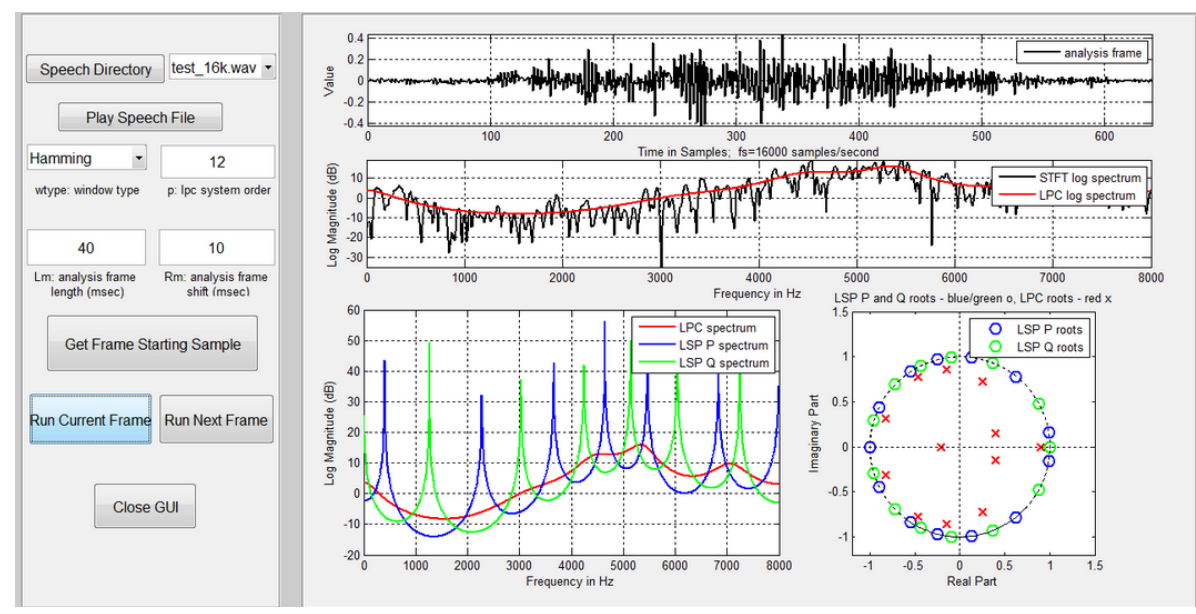

Fig 4: Simulation of proposed Speech Recognition in Mobile Customer Care Service

\section{Conclusion}

It can be concluded that in the push button IVR method, the customers has to wait for some time to get their required service. By using speech recognition in mobile customer care services, the process can be faster considering the other normal IVR techniques. Here the Speech is recognized using Knn algorithm by the matlab simulations. In Future enhancement, the customer care model will be implemented with Zigbee module and microcontroller. Then the audio output of customer request will be delivered back to the customer. So the customers need not want to wait for long time and the information can be received immediately.

\section{References}

[1] P. Ding, L. He, X. Yan, R. Zhao, and J. Hao,2008, "Robust mandarin speech recognition in car environments for embedded navigation system," IEEEon Consumer Electronics, vol. 54, no. 2, pp. 584-590

[2] Y. Oh, J. Yoon, J. Park, M. Kim, and H. Kim, “A name recognition based call-and-come service for home robots,” IEEE Trans. On Consumer Electronics, vol. 54, no. 2, pp. 247-253, 2008 
[3] O. Hazrati, S. Ghaffarzadegan and J. H. L. Hansen, "Leveraging automatic speech recognition in cochlear implants for improved speech intelligibility under reverberation," 2015 IEEE International Conference on Acoustics, Speech and Signal Processing (ICASSP), Brisbane, QLD, 2015, pp. 50935097, doi: 10.1109/ICASSP.2015.7178941.

[4] L. Pfeifenberger, T. Schrank, M. Zohrer, M. Hagmüller and F. Pernkopf, "Multi-channel speech processing architectures for noise robust speech recognition: 3rd CHiME challenge results," 2015 IEEE Workshop on Automatic Speech Recognition and Understanding (ASRU), Scottsdale, AZ, 2015, pp. 452-459, doi: 10.1109/ASRU.2015.7404830.

[5] V. Mitra et al., "Improving robustness against reverberation for automatic speech recognition," 2015 IEEE Workshop on Automatic Speech Recognition and Understanding (ASRU), Scottsdale, AZ, 2015, pp. 525-532, doi: 10.1109/ASRU.2015.7404840.

[6] E. Messner, H. Pessentheiner, J. A. Morales-Cordovilla and M. Hagmüller, "Adaptive differential microphone arrays used as a front-end for an automatic speech recognition system," 2015 IEEE International Conference on Acoustics, Speech and Signal Processing (ICASSP), Brisbane, QLD, 2015, pp. 2689-2693, doi: 10.1109/ICASSP.2015.7178459.

[7] Y. Iribe, N. Kitaoka and S. Segawa, "Development of new speech corpus for elderly Japanese speech recognition," 2015 International Conference Oriental COCOSDA held jointly with 2015 Conference on Asian Spoken Language Research and Evaluation (O-COCOSDA/CASLRE), Shanghai, 2015, pp. 2731, doi: 10.1109/ICSDA.2015.7357859.

[8] J. Chaloupka, J. Nouza, J. Malek and J. Silovsky, "Phone speech detection and recognition in the task of historical radio broadcast transcription," 2015 38th International Conference on Telecommunications and Signal Processing (TSP), Prague, 2015, pp. 1-4, doi: 10.1109/TSP.2015.7296399.

[9] N. Ruiz and M. Federico, "Phonetically-oriented word error alignment for speech recognition error analysis in speech translation," 2015 IEEE Workshop on Automatic Speech Recognition and Understanding (ASRU), Scottsdale, AZ, 2015, pp. 296-302, doi: 10.1109/ASRU.2015.7404808.

[10] D. Baby, T. Virtanen, J. F. Gemmeke and H. Van hamme, "Coupled Dictionaries for Exemplar-Based Speech Enhancement and Automatic Speech Recognition," in IEEE/ACM Transactions on Audio, Speech, and Language Processing, vol. 23, no. 11, pp. 1788-1799, Nov. 2015, doi: 10.1109/TASLP.2015.2450491. 